\title{
UV Light Generation and Challenging Photoreactions \\ Enabled by Upconversion in Water
}

\author{
Björn Pfund ${ }^{a}$, Debora M. Steffen ${ }^{a}$, Mirjam R. Schreier ${ }^{a}$, Maria-Sophie Bertrams $^{a}$, Chen Ye $^{b}$, Karl $^{2}$ \\ Börjesson $^{\mathrm{b}}$, Oliver S. Wenger ${ }^{\mathrm{a} *}$ and Christoph Kerzig ${ }^{\mathrm{a} *}$
}

\begin{abstract}
Sensitized triplet-triplet annihilation (sTTA) is the most promising mechanism for pooling the energy of two visible photons, but its applications in solution were so far limited to organic solvents, with a current maximum of the excited-singlet state energy of $3.6 \mathrm{eV}$. By combining tailor-made iridium complexes with naphthalenes, we demonstrate blue-light driven upconversion in water with unprecedented singlet-state energies approaching $4 \mathrm{eV}$. The annihilators have outstanding excited-state reactivities enabling challenging photoreductions driven by STTA. Specifically, we found that an aryl-bromide bond activation can be achieved with blue photons, and we obtained full conversion for the very energy-demanding decomposition of a persistent ammonium compound as typical water pollutant, not only with a cw laser but also with an LED light source. These results provide the first proof-of-concept for the usage of low-power light sources for challenging reactions employing blue-to-UV upconversion in water, and pave the way for the further development of sustainable light-harvesting applications.
\end{abstract}

\section{Introduction}

Sensitized triplet-triplet annihilation (sTTA), ${ }^{1}$ also called triplet fusion, is a powerful mechanism for constructing light-harvesting systems, comprising a sensitizer (donor) and an annihilator (acceptor). ${ }^{2-6}$ The sensitizer triplets produced by low-energy photons undergo a donor-to-acceptor triplet-triplet energy transfer (TTET) followed by a TTA between two acceptor triplets, yielding an emissive high-energy singlet state of the acceptor (upper left part of Fig. 1). As a result of the different mechanistic functions of sensitizers (light absorption and energy transfer) and annihilators (production of excited-singlet states via annihilation) in such a system,

${ }^{[a]}$ B. Pfund, D. M. Steffen, M. R. Schreier, M.-S. Bertrams, O. S. Wenger, C. Kerzig Department of Chemistry, University of Basel, St. Johanns-Ring 19, 4056 Basel (Switzerland)

${ }^{\text {[b] }}$ C. Ye, K. Börjesson Department of Chemistry and Molecular Biology, University of Gothenburg, Kemigården 4, 41296 Gothenburg (Sweden)

E-mail: christoph.kerzig@unibas.ch, oliver.wenger@unibas.ch 
largely individual optimization of each component is readily possible. This allows employing the STTA mechanism for several light-driven tasks beyond its initial photon upconversion (lighting) use, including visible-- ${ }^{7-9}$ or even NIR-light ${ }^{10}$ driven photo(redox) reactions, and biochemical, ${ }^{11-13}$ photochromic $^{14,15}$ as well as photovoltaic ${ }^{16}$ applications. The modularity of this two-component system, combined with the feasibility of biphotonic (i.e., two-photon) mechanisms at noncoherent low photon fluxes, ${ }^{4}$ contributes to the ongoing popularity of sTTA.

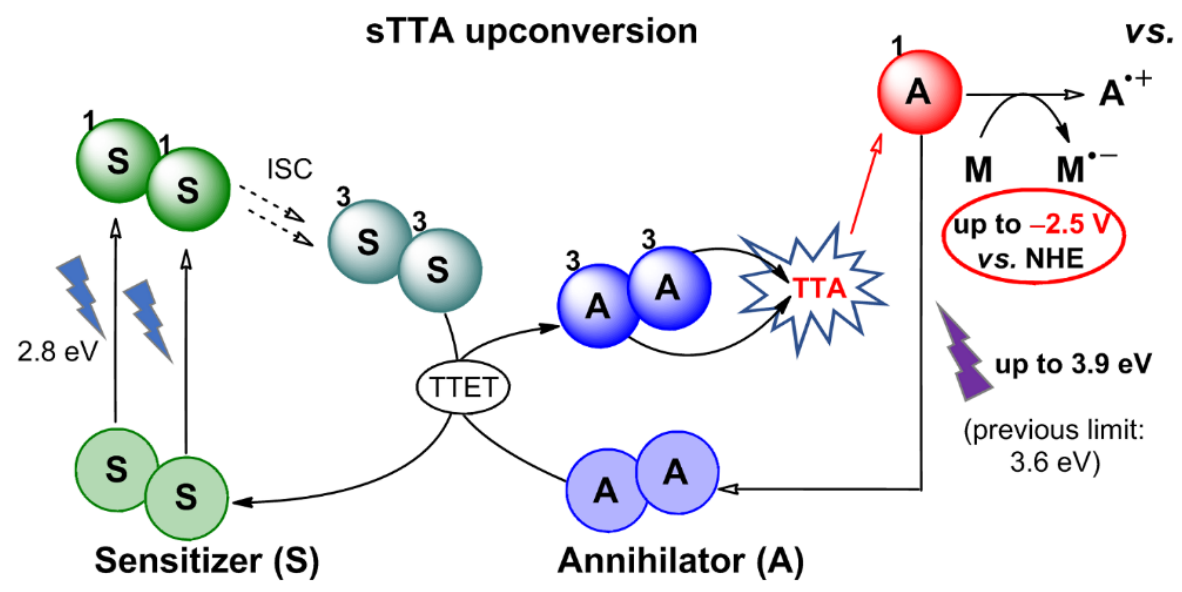

vs. direct catalyst excitation

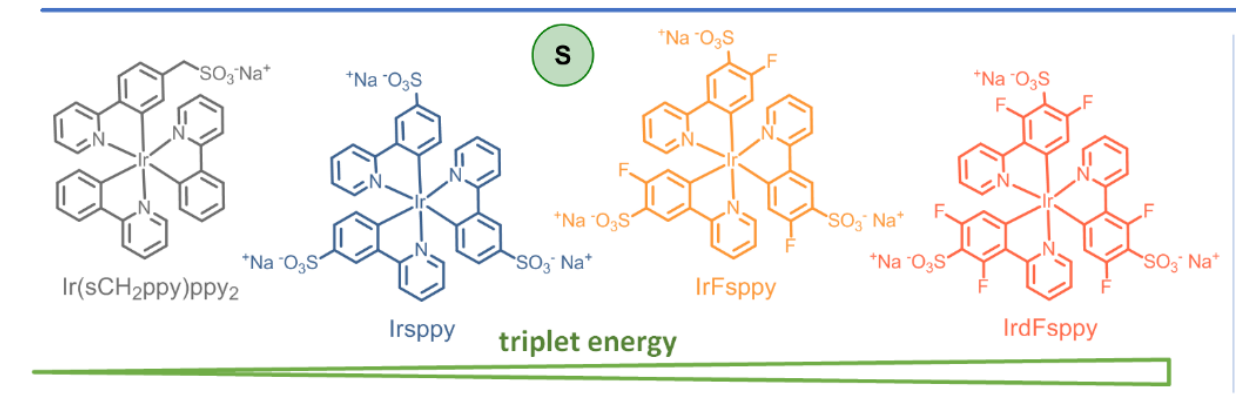

Photocatalyst (PC)

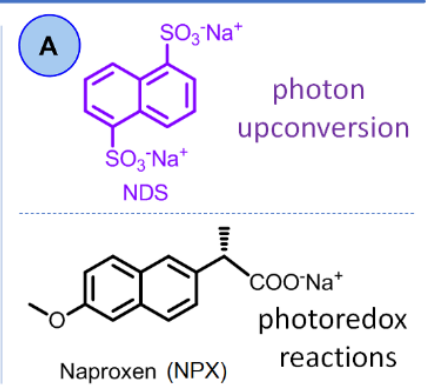

Figure 1. Aqueous blue-to-UV upconversion strategy. Upper part, simplified sTTA mechanism and its use for blue-to-UV upconversion in water as well as challenging photoredox reactions (left) that are impossible through conventional catalyst excitation with a single visible photon in aqueous solution (right). Lower part, molecular structures and abbreviations of the water-soluble sensitizers (left, with increasing triplet energies) and acceptors/annihilators (right) investigated in this work. For further explanations, see text and SI.

Owing to solubility and reactivity issues, examples of TTA-based upconversion in pure water are extremely scarce. ${ }^{17,18}$ The same holds true for visible-light driven sTTA systems (in organic solvents) enabling high-energy UV emission below $350 \mathrm{~nm},{ }^{19,20}$ where the intensity of the terrestrial solar spectrum sharply drops. The challenging combination of both - Vis-to-UV upconversion in the green solvent water ${ }^{21}$ - would represent a substantial step towards sustainable solar light-driven systems for energy-demanding photochemical processes, given 
the current rethinking of replacing organic solvents by aqueous systems. ${ }^{22-27}$

Aiming to harvest a larger part of the solar spectrum, many scientists currently focus on red/NIR-to-visible upconversion, ${ }^{11,28-31}$ Thermodynamically, these strategies cannot be exploited for chemical reactions requiring more energy input than a single visible photon. However, visible-to-UV upconversion provides an attractive alternative to direct UV excitation, which is not only harmful and unsustainable when produced through mercury-based light sources, but it suffers also from selectivity and filter effect issues. ${ }^{32}$ To date, the most highly energetic excited annihilator singlets produced through sTTA with visible light as input have maximum energies of about $3.6 \mathrm{eV}^{20,33-36}$ corresponding to $345 \mathrm{~nm}$. Visible-to-UV upconversion has the potential to convert readily available blue or violet photons to UVB (280-315 nm) light, but this is heavily underexplored. Herein, we demonstrate that the combinations of tailor-made water-soluble sensitizers with selected naphthalene derivatives result in novel aqueous STTA systems. These systems are capable of providing unprecedented delayed emission photon energies or redox potentials for energy-demanding reductions that cannot be obtained with conventional (visible) one-photon excitation ${ }^{32}$ (Fig. 1). With detailed mechanistic investigations and lab-scale photoredox applications, which would otherwise require more aggressive species usually produced at much higher photon fluxes per area, ${ }^{37-39}$ we show that STTA upconversion in pure water has the potential to be developed into a "green" tool in chemistry.

\section{Results and discussion}

Building on our recently developed complex Irsppy (see Fig. 1 for its structure), ${ }^{25,37,39}$ which is a water-soluble version of the popular photosensitizer fac-Ir(ppy $)_{3}$, we prepared and characterized three novel water-soluble Ir-based complexes. Guided by the structure-property relations for conventional photoactive Ir complexes, ${ }^{40}$ water-soluble sensitizers were synthesized with particular emphasis on increasing the triplet energy to improve the TTET reactivity accordingly. The photochemical properties of the novel complexes were investigated in detail and compared to Irsppy (Section 2.1). Synthetic procedures and characterization data are in the Supporting Information (SI, Section S2).

The previous observation of highly energetic delayed emission caused by TTA upon direct 
excitation of a naphthalene derivative in neat water ${ }^{41}$ prompted us to test several water-soluble annihilator candidates (naphthalene, $p$-terphenyl and biphenyl derivatives), in combination with the above-mentioned sensitizers. Pertinent properties of the best-suited annihilators, which do not suffer from solubility problems ${ }^{18}$ and excimer formation issues ${ }^{42}$ (see Section S8 of the SI for details), are outlined in Section 2.2, and the structures of the main compounds used to achieve blue-to-UV upconversion are displayed in the lower part of Fig. 1.

Detailed spectroscopic studies (see SI for method descriptions and details) that substantiate the STTA upconversion mechanism with the annihilator possessing the most highly energetic excited-singlet state ( 3.9 eV) are presented in Section 2.3, whereas our key findings on sTTAdriven photoreductions employing the annihilator with the most negative excited-state oxidation potential (about $-2.5 \mathrm{~V}$ vs. NHE) can be found in Section 2.4.

\subsection{Sensitizer molecules and their properties}

Besides water solubility, visible-light absorptivity and high intersystem crossing (ISC) quantum yields, sufficiently long triplet lifetimes and high triplet energies are the basic requirements for the sensitizers. The latter should be at least on the order of $2.6 \mathrm{eV}^{41,43}$ to efficiently sensitize the annihilators under study, thereby ideally achieving blue-to-UV upconversion in water. Watersoluble photoactive compounds with such properties are scarce; we therefore prepared three new Ir complexes and compared their photophysical properties with those of Irsppy.

UV-Vis absorption spectra, steady-state emission spectra, and kinetic emission measurements for all sensitizers in alkaline water are shown in Fig. 2, along with the corresponding triplet state energies $\left(E_{\mathrm{T}}\right)$ and lifetimes $\left(\tau_{0}\right)$. Basic $\mathrm{pH}$ conditions were used throughout this study to ensure good solubility of annihilators (Section 2.2) and substrates (Section 2.4). In Fig. 2, the sensitizers are arranged such that their triplet energies increase from the top to the bottom of the figure. The novel monoanionic complex with one (slightly) electron-withdrawing group only, $\operatorname{Ir}\left(\mathbf{s C H}_{2}\right.$ ppy)ppy (see Fig. 1 for its structure), has a triplet energy identical to that of its lipophilic congener $\operatorname{Ir}(\mathrm{ppy})_{3}(2.50 \mathrm{eV}){ }^{44}$ which is significantly lower than the Irsppy triplet energy (2.65 $\mathrm{eV}$ ). Further reduction of the electron density at the cyclometalated ligands of the Irsppy structure by fluorination ${ }^{40}$ results in even higher triplet energies, as is immediately evident from the blue-shifted emission when going from Fig. $2 b$ to Fig. $2 c / d$. The observed triplet energy 
increase is accompanied by a shift of the long-wavelength UV-Vis absorption band (i.e., the MLCT absorption) $)^{37,45}$ towards the UV. However, even IrdFsppy, which has a triplet energy as high as $2.81 \mathrm{eV}$, noticeably absorbs at our excitation wavelengths employed for photon upconversion experiments (447 nm) and STTA-driven photoredox reactions (447 $\mathrm{nm}$ or $455 \mathrm{~nm}$ ). All photosensitizers under study are expected to undergo ultrafast and quantitative ISC, ${ }^{37}$ and they possess triplet lifetimes of about $2 \mu$ s (Fig. 2), which is long enough for efficient bimolecular reactions.

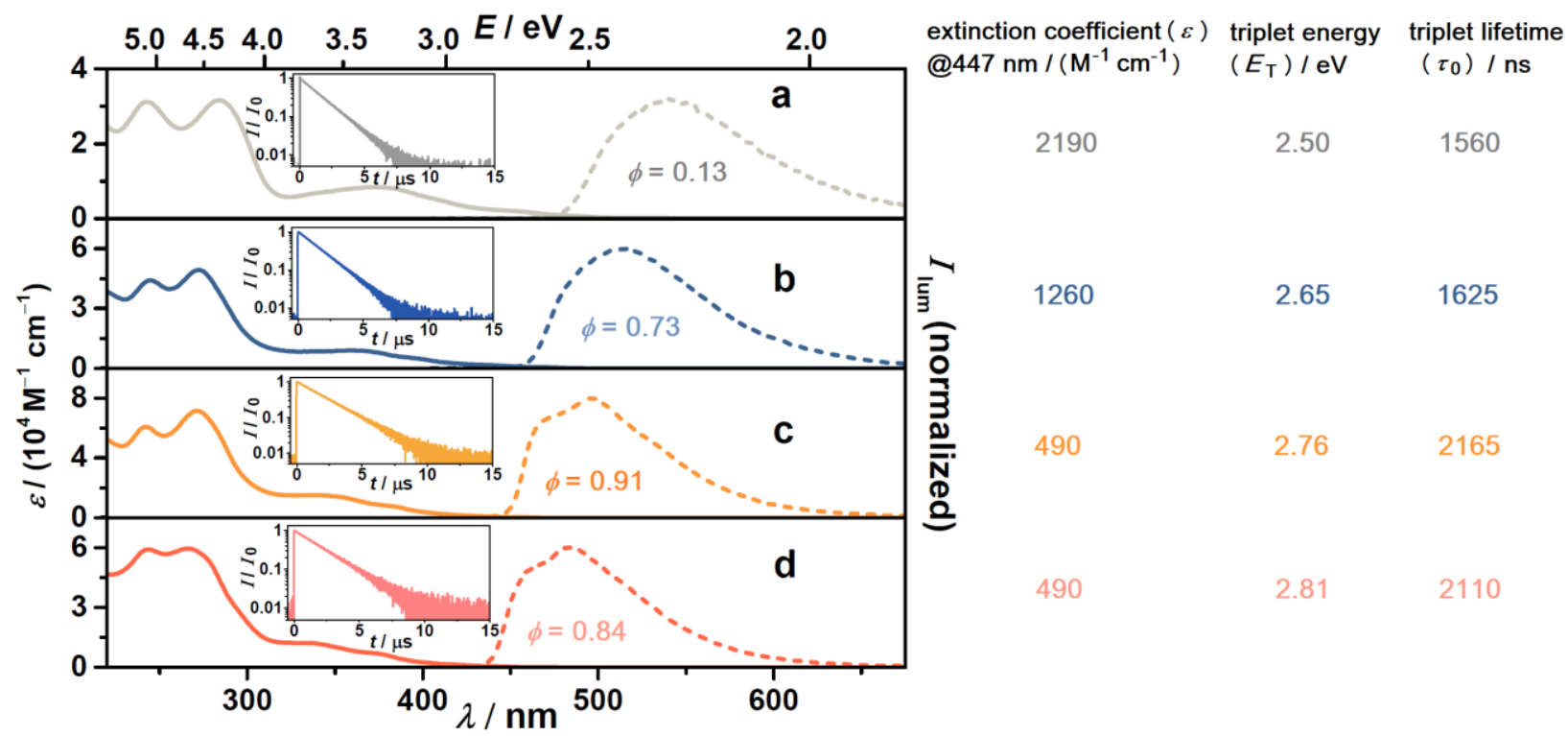

Figure 2. Sensitizer characteristics. Experimental data summarizing the pertinent ground- and excitedstate properties of the Ir-based sensitizers (a, $\operatorname{Ir}\left(\mathbf{s C H}_{2}\right.$ ppy)ppy ${ }_{2} ; \mathrm{b}$, Irsppy; c, IrFsppy; d, IrdFsppy). Main plots, calibrated absorption (solid lines) and normalized luminescence (dashed lines) spectra obtained in aqueous solutions containing $50 \mathrm{mM} \mathrm{NaOH}$. Insets, normalized kinetic emission traces of the sensitizers upon $420 \mathrm{~nm}(14 \mathrm{~mJ})$ excitation of strongly diluted ( 30 $\mu \mathrm{M})$ Ar-saturated dye solutions with laser pulses of $\sim 10$ ns duration. For further details, see text and Section S1 of the SI.

The photochemical characteristics of the Ir complexes summarized in Fig. 2 prompted us to compare their performance in a triplet-sensitized lab-scale isomerization reaction. ${ }^{46}$ To this end, trans-3-fluorocinnamate (10 $\mathrm{mM}$ in aq. $\mathrm{NaOH}$ ) with a triplet energy of $\sim 2.4 \mathrm{eV}^{39}$ was irradiated with a blue light source $(447 \mathrm{~nm}, 1 \mathrm{~W})$ in the presence of $0.3 \mathrm{~mol} \%$ of the Ir-catalysts, and the composition of the respective photostationary state was analyzed. In line with TTET reaction pathways and the sensitizer triplet energies in Fig. 2 (for further explanations, see SI, Section S3.2), we obtained yields of the desired cis isomer between $55 \%$ and $90 \%$, corresponding to 
turn-over numbers (TONs) up to 300. With future photoredox applications of our novel watersoluble Ir complexes in mind, we determined their excited-state oxidation potentials and observed tunability between -1.5 and -1.7 V vs. NHE (SI, Section S2.1).

Laser flash photolysis studies on the TTET between excited Ir complexes and potential annihilators revealed ${ }^{3} \operatorname{Ir}\left(\mathbf{s C H}_{2} \mathbf{p p y}\right) \mathbf{p p y} \mathbf{y}_{2}$ to be too low in energy for efficient sensitization, whereas all other complexes gave positive results (Section 2.3). Among these three potent sensitizers, Irsppy and IrFsppy are ideal candidates for the upconversion applications presented in Sections 2.3 and 2.4, because of their long-term inherent photostability under conditions of intense blue-light irradiation (details are given in the SI, Section S3.1).

\subsection{Acceptors/annihilators and their properties}

Several tested cationic annihilators (SI, Section S8), which are themselves highly water-soluble, could not be combined with the anionic sensitizers presented in Section 2.1. Coulombic interactions provide a plausible explanation: the opposite-charged ionic species aggregate in water, thereby causing both solubility problems and static quenching effects. These detrimental effects are completely absent for the anionic acceptors/annihilators, whose photochemical properties of the respective ground states and emissive excited-singlet states are depicted in Fig. 3. Interestingly, recent upconversion studies employed naphthalene derivatives merely as energy transfer mediators but not as annihilators. ${ }^{34,47,48}$ Analyzing the intersections of absorption and emission spectra as shown in Fig. 3, we determined excited-singlet state energies $\left(E_{00}\right)$ of $\sim 3.9 \mathrm{eV}$ and $\sim 3.7 \mathrm{eV}$ for 1,5-naphthalenedisulfonate (NDS) and the carboxylate of the painkiller naproxen (NPX), respectively. These energies compare favorably to those of the most highly energetic singlet states previously produced through sTTA $(\sim 3.6 \mathrm{eV}) .{ }^{20,33,34}$ With excited-singlet state lifetimes approaching 10 ns (insets in Fig. 3), both naphthalene derivatives are not ultra-short lived, laying the grounds for UC-driven bimolecular substrate activation reactions initiated by the singlet-excited annihilators $\left({ }^{1} A^{*}\right)$ in water. The emission quantum yield of the highly-energetic excited NDS singlet, which is most promising for blue-to-UV photon upconversion, is largely $\mathrm{pH}$-independent and has been determined to be $0.14 \pm 0.01$ (SI, Section S4). 


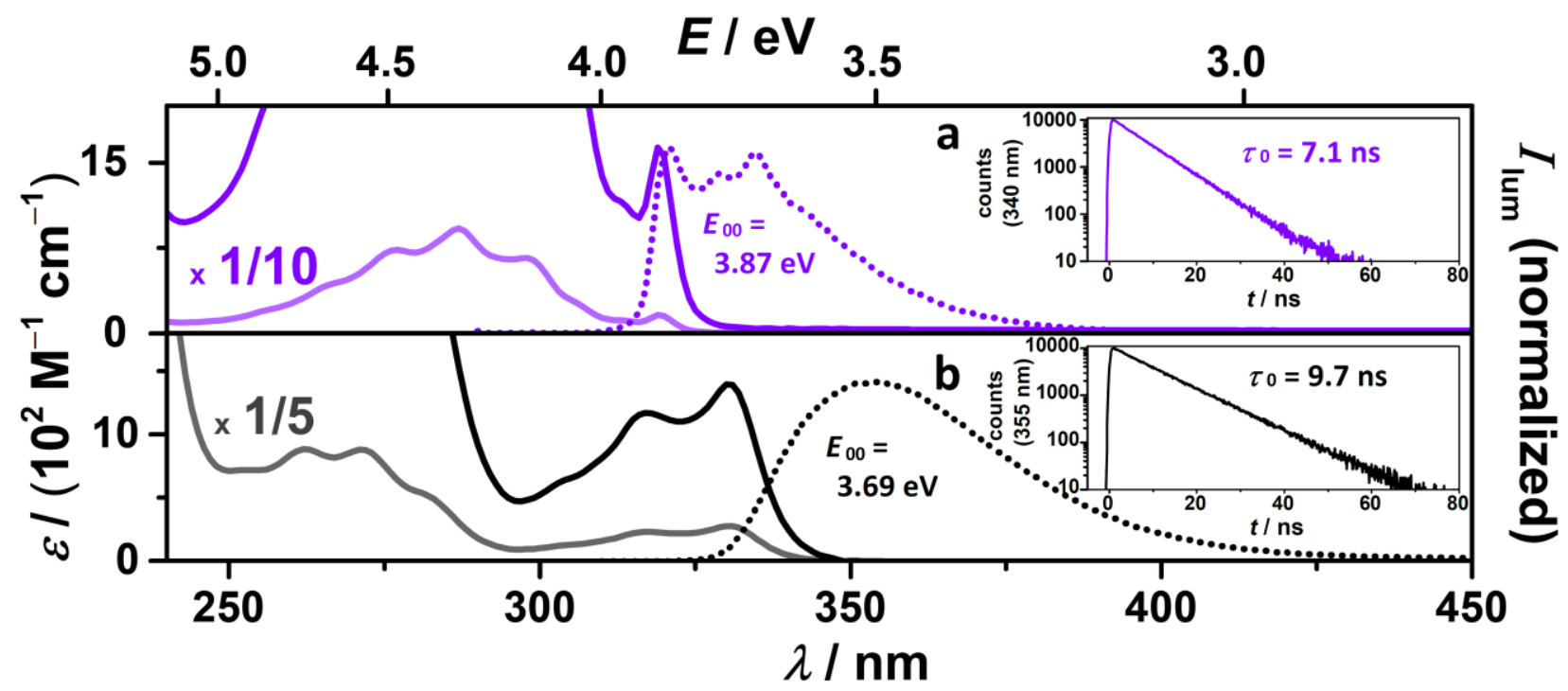

Figure 3. Photophysical properties of the acceptors/annihilators under study (a, NDS; b, NPX). Main plots, calibrated absorption (solid lines) and normalized emission (dotted lines) spectra obtained in aqueous solutions containing $50 \mathrm{mM} \mathrm{NaOH}$. For clarity, the absorption spectra are displayed with and without scaling factors. Insets, excited-singlet state lifetimes measurements using the TCSPC technique (excitation wavelength, $320 \mathrm{~nm}$ ) in de-oxygenated solution with naphthalene concentrations of about $0.2 \mathrm{mM}$.

The triplet energy of NDS is $2.58 \mathrm{eV},{ }^{41}$ and given the negligible effect of several completely different substituents on the triplet energy of the naphthalene chromophore ${ }^{43}$ (triplet energy of unsubstituted naphthalene, $2.64 \mathrm{eV}$ ), we expect a similar triplet energy for NPX. The established extinction coefficient $(\varepsilon)$ of ${ }^{3} \mathrm{NDS}^{41}$ allowed us to analyze its mixed first- and second order decay kinetics upon direct NDS excitation (followed by ISC). A natural triplet lifetime as long as $1.1 \mathrm{~ms}$ and a self-quenching (the reaction of two triplets, which proceeds via TTA as shown in Section 2.3) rate constant of $1.1 \times 10^{9} \mathrm{M}^{-1} \mathrm{~s}^{-1}$ were obtained in alkaline water at room temperature (see Section S4 of the SI for details). Although a recent report describes the photodecomposition of NPX in phosphate-buffered water-acetonitrile mixtures, ${ }^{49}$ we did not observe any stability problems of excited NPX in our reaction medium (neat water at $\mathrm{pH}>12$ ), neither upon direct NPX excitation nor after sensitization. Regarding the ability of the singlet-excited naphthalenes for direct substrate activations by one-electron reductions, the excited-state oxidation potentials $\left(E_{1 / 2}\left(\mathrm{~A}^{\bullet+} /{ }^{1} \mathrm{~A}^{*}\right)\right)$ of both derivatives are the key parameters. Taking the (ground state) oxidation potentials $s^{41,50}$ and the $E_{00}$ energies of the naphthalenes (Fig. 3), one can estimate $E_{1 / 2}\left(\mathrm{~A}^{\bullet+} /{ }^{1} \mathrm{~A}^{*}\right)$ values of $-1.9 \mathrm{~V}(\mathrm{NDS})$ and $-2.5 \mathrm{~V}(\mathrm{NPX})$, both vs. NHE. Thus, the replacement of two electron-withdrawing substituents (NDS) by one electron-donating substituent and one 
essentially "inactive" substituent (NPX) greatly facilitates the oxidation of the naphthalene chromophore, thereby increasing the excited-singlet state reactivity significantly. This pronounced effect clearly overcompensates the lower excited-singlet state energy of NPX.

\subsection{Blue-to-UV photon upconversion in water}

Evidently the triplet of NDS is accessible via sensitization as the comparison of the transient absorption spectra shown in Fig. 4a (sensitization with Irsppy) and Fig. 4b (direct NDS excitation and ISC) reveals - both spectra are virtually identical and in agreement with the known absorption spectrum of ${ }^{3}$ NDS. ${ }^{41}$ The delayed emission spectrum in Fig. $4 \mathrm{~b}$ (inset) was recorded with a time-delay long enough to ensure complete singlet-excited state decay after direct laser excitation. It shows the emission band of ${ }^{1}$ NDS* providing clear evidence that ${ }^{3}$ NDS undergoes TTA. (There is a slight filter effect at the blue edge of the spectrum in Fig. $4 \mathrm{~b}$ compared to the spectrum displayed in Fig. 3a, which was measured in more dilute solution.) The same spectroscopic signatures of ${ }^{1}$ NDS* $^{*}$ were observed in the delayed emission spectra in Fig. 4a upon blue light excitation of our Ir complexes capable of sensitizing ${ }^{3}$ NDS. In addition to the ${ }^{1}$ NDS* emission band, two other features can be observed in the delayed emission spectra of Fig. 4a, namely, a weak emission band/shoulder centered at about $400 \mathrm{~nm}$ and intense emission in the green spectral region with a short wavelength edge at $\sim 435 \mathrm{~nm}$. The former is most likely due to excimer emission ${ }^{42}$ caused by quenching of ${ }^{1}$ NDS* by NDS ground state molecules, which were present in 100-times higher concentrations than in the series of experiments presented in Fig. 4b, whereas the green luminescence (with an onset at $435 \mathrm{~nm}$ ) originates from delayed sensitizer emission due to reverse $\mathrm{TTET}^{51}$ from ${ }^{3}$ NDS to the respective Ir sensitizer ground state.

The simultaneous monitoring of sensitizer emission quenching (Fig. 4c) and ${ }^{3}$ NDS formation (Fig. 4d) under standardized conditions (identical sensitizer absorption at our excitation wavelength

and $15 \mathrm{mM}$ of NDS) provided further insights into the sensitization process. First, ${ }^{3}$ NDS is produced (monitored by transient absorption measurements at $440 \mathrm{~nm}$ ) with the same rate as the respective triplet of the Ir sensitizer is quenched, confirming that the desired TTET is taking place. Second, the fluorinated sensitizers with their higher triplet energies (compare, Fig. 2) transfer the energy more rapidly than Irsppy, which is in line with a higher driving force for the TTET event. Third, the observed TTET efficiencies are between $84 \%$ and $95 \%$ (more details on 
the TTET kinetics, including detailed Stern-Volmer quenching studies, can be found in Section S5 of the SI), confirming the efficient use of the photons absorbed by the sensitizers for ${ }^{3}$ NDS generation under our conditions. Additional transient absorption experiments on a much longer timescale (Fig. 4e) reveal that the visible-light produced NDS triplets completely decay within $\sim 1$ ms. The ${ }^{3}$ NDS decay in the sensitizer-containing systems (Fig. 4e) is significantly faster than upon direct ${ }^{3}$ NDS production with $320 \mathrm{~nm}$ laser pulses (SI, Fig. S6), which is in line with the abovementioned reverse TTET as an additional ${ }^{3}$ NDS deactivation channel. Furthermore, comparative time-resolved emission and absorption investigations ${ }^{52}$ with the NDS / Irsppy system (SI, Fig. S7) permit the unambiguous observation of this reverse TTET process.

Blue-to-UV photon upconversion in water is not limited to pulsed laser excitation, as our investigations with an inexpensive continuous wave $(\mathrm{cw})$ excitation light source (447 $\mathrm{nm}$, up to 1 W optical output, the output power has to be multiplied by $8 \mathrm{~cm}^{-2}$ for its conversion to power density, see page S3 for details) establish. Figs. 4f-g display power-dependent results on the upconverted emission, either measured at low sensitizer concentrations to obtain evidence for the two-photon nature of the mechanism (Fig. 4f), or at a much higher concentration to reach the linear regime of the UC power dependence (Fig. $4 \mathrm{~g})^{53}$.

Fig. $4 \mathrm{f}$ illustrates steady-state emission spectra of the NDS / IrFsppy UC system; a finestructured band with maxima at $321 \mathrm{~nm}, 330 \mathrm{~nm}$ and $335 \mathrm{~nm}$, very similar to the emission spectrum upon direct NDS excitation (Fig. 3a), can be observed, whereas control experiments without NDS or IrFsppy do not yield any signals in the $300-400 \mathrm{~nm}$ spectral region. Comparing the energy of the short-wavelength maximum of the upconverted emission (321 nm) with that of the blue photons (447 $\mathrm{nm}$ ) used for excitation, we got an anti-Stokes shift as high as $1.09 \mathrm{eV}$. The fit of the upconverted emission dependence on the $\mathrm{cw}$ laser output with a power function yielded an exponent of 1.9 (inset of Fig. 4f, violet data set) confirming the biphotonic UC mechanism. In contrast to this quadratic power dependence, a linear power dependence has been obtained for the emission of ${ }^{3}$ IrFsppy produced upon (monophotonic) IrFsppy excitation (inset of Fig. 4f, orange data set). 

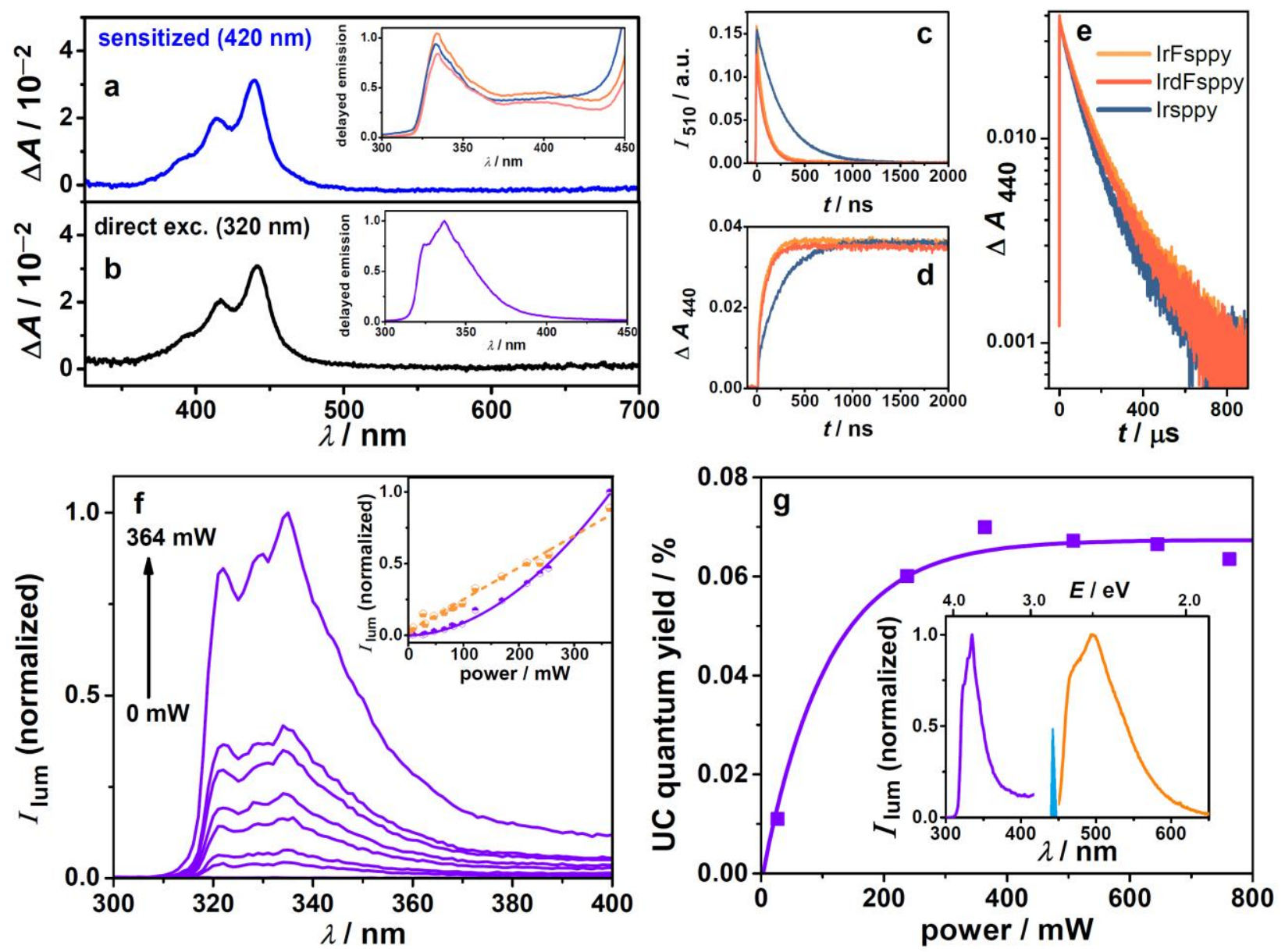

Figure 4. Spectroscopic investigations on the upconversion mechanism. a,c-e, Sensitized generation of the triplet state of NDS ( ${ }^{3} \mathrm{NDS}$ ) in alkaline Ar-saturated water (with $22 \mu \mathrm{M}$ Irsppy, $30 \mu \mathrm{M}$ IrFsppy, or 41 $\mu \mathrm{M}$ IrdFsppy and $15 \mathrm{mM}$ NDS excited at $420 \mathrm{~nm}$ with $12 \mathrm{~mJ}$ laser pulses). b, Direct generation of ${ }^{3}$ NDS ( $0.15 \mathrm{mM}$ NDS excited at $320 \mathrm{~nm}$ with $1.2 \mathrm{~mJ}$ laser pulses). a-b, Transient absorption spectra after sensitizer (a) or singlet-excited NDS (b) decay time-integrated over 200 ns. The insets display delayed emission spectra time-integrated over $1 \mathrm{~ms}$ (a) or $2 \mathrm{~ms}$ (b). c-e, Kinetic traces of the triplet sensitizer quenching via TTET (c), ${ }^{3}$ NDS formation (d) and the decay of sensitized ${ }^{3}$ NDS (e). f-g, Blue-to-UV upconversion using a cw light source for excitation at $447 \mathrm{~nm}$. $\mathrm{f}$, Quadratic power dependence of the upconverted emission (violet) in the system IrFsppy (30 $\mu \mathrm{M})$ / NDS (5 mM) with the linear dependence of the unquenched sensitizer emission for comparison (inset). g, Quantum yield determinations at higher IrFsppy $(135 \mu \mathrm{M})$ and NDS (15 $\mathrm{mM})$ concentrations. The inset shows normalized upconverted emission (violet) and reference signals (orange, unquenched IrFsppy emission), together with the output spectrum of the blue cw laser.

The STTA-UC quantum yield determinations presented in Fig. $4 \mathrm{~g}$ were carried out relative to the unquenched sensitizer emission as reference (details are given in the SI, Section S6), and the quantum yields so obtained were normalized to a theoretical limit of $100 \%$. The maximum UC quantum yield for the NDS / IrFsppy system is estimated to be $\sim 0.07 \%$. A similar series of 
experiments with the NDS / Irsppy system gave a slightly lower quantum yield ( 0.05\%, Fig. S10 of the $\mathrm{SI}$ ), in line with the UC efficiency differences of both systems under pulsed laser excitation conditions (Fig. 4a, inset). These quantum yields seem modest at first glance, but also in organic solvents most of the reported blue-to-UV UC systems are highly inefficient. ${ }^{34}$ Moreover, given the moderate emission quantum yield of NDS (0.14, see Section 2.2), which limits the photon upconversion efficiency, the actual yields of high-energy singlet-excited naphthalenes available for challenging redox chemistry are more than seven times ( 1 divided by 0.14 ) higher than the estimated photon upconversion quantum yields.

The results presented in this section unambiguously establish that blue-to-UV UC via the STTA mechanism is feasible in pure water. Gratifyingly, the investigated aqueous Ir sensitizer / NDS UC systems can compete with the most efficient reported STTA-UC systems in organic solvents in terms of anti-Stokes shift, ${ }^{28,29,54}$ and they outperform the highest anti-Stokes shift previously observed for a blue-to-UV UC system. ${ }^{20}$

Comparable results on the aqueous sTTA mechanism were obtained with NPX instead of NDS as acceptor/annihilator (see SI, Section S6). The main difference to the UC investigation of Fig. 4 (with NDS) is the considerably lower anti-Stokes shift for the Ir sensitizer / NPX systems $(\sim 0.7 \mathrm{eV})$. However, owing to the promising redox properties of singlet excited NPX, these UC systems are well suited for driving challenging reductions with blue photons, as we will show in the following section.

\subsection{Lab-scale photoredox applications}

Exploiting the sTTA mechanism to generate highly reactive species with low energy photons is an emerging approach to perform challenging photoreactions under mild conditions using common LEDs or inexpensive diode lasers as light sources. ${ }^{7-10,18}$ In our specific example with blue light as energy input, the two-photon approach (sTTA) allows us to obtain redox potentials up to $0.8 \mathrm{~V}$ higher than those accessible with the already highly reducing $\mathrm{Ir}$ complexes. The results of this section demonstrate that this reactivity boost can be exploited for driving energydemanding reactions via blue-to-UV UC in water, which would otherwise require more aggressive conditions or much longer reaction times.

First, we turned to the activation of a $\mathrm{C}-\mathrm{Br}$ bond and we selected the substrate 4-bromo-2- 
chloro-5-fluorobenzoate for mechanistic studies, whose reaction with excited Irsppy is endergonic resulting in extremely low debromination rates. ${ }^{39}$ Efficient debromination to give 2chloro-5-fluorobenzoate in 63\% yield was observed with the Irsppy / NPX UC system (left part of Fig. 5), which evidently produces the highly reducing ( $-2.5 \mathrm{~V}$ vs. NHE) singlet-excited state of NPX ( ${ }^{1}$ NPX*) upon $447 \mathrm{~nm}$ excitation (see SI, Fig. S10). A Stern-Volmer analysis for the quenching of ${ }^{1}$ NPX* by 4-bromo-2-chloro-5-fluorobenzoate gave a diffusion-controlled rate constant for the bimolecular substrate activation step and subsequent bromide ion release (right part of Fig. 5). In line with the 10,000 -times slower substrate activation rate constant for ${ }^{3}$ Irsppy, ${ }^{39}$ we observed only traces of the hydrodehalogenation product during control irradiation experiments without NPX. Full particulars of all preparative-scale irradiation experiments and product/starting material analyses can be found in Section S7 of the SI. The hydrogen donor isopropanol has been added to the reaction mixture to ensure efficient interception of the reactive sigma radical produced by substrate reduction, which avoids side products. Interestingly, quantitative NMR investigations revealed that isopropanol acts not only as hydrogen atom donor but also as electron donor. The dimethyl ketyl radical (or its radical anion) generated upon hydrogen abstraction is highly reducing ${ }^{38}$ and clearly capable of regenerating oxidized NPX. In direct consequence, the reaction shown in Fig. 5 (left part) consumes only minor amounts of NPX. As opposed to the first and only other aqueous UCdriven photoredox application, ${ }^{18}$ our system (Fig. 5) is thus catalytic regarding both the sensitizer (TON 100) and the annihilator (TON 15). 
$\overbrace{F}^{c l} c 00$
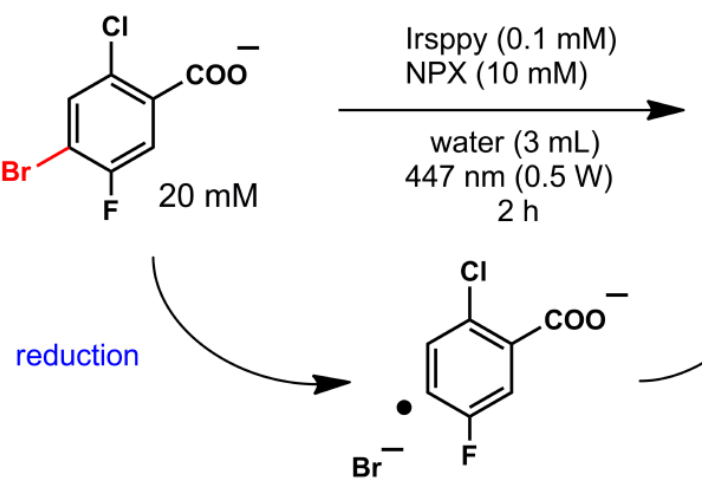
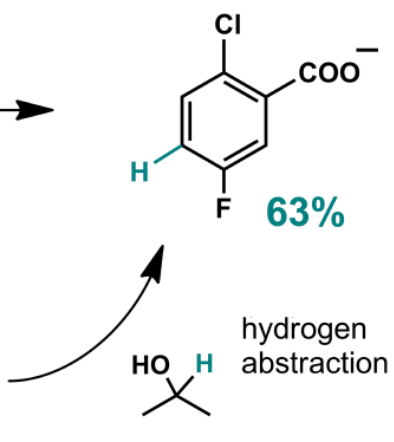

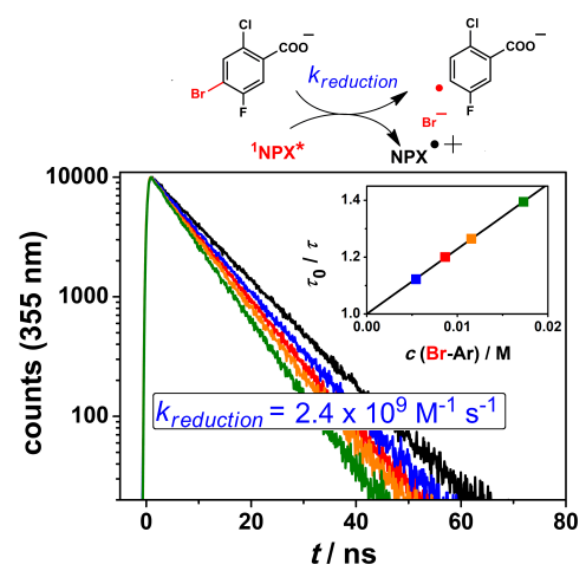

Figure 5. Reductive debromination via upconversion. Conversion of 4-bromo-2-chloro-5-fluoro-benzoate in Ar-saturated aqueous solution by singlet-excited naproxen (NPX) produced through sTTA using a blue $(447 \mathrm{~nm}) \mathrm{cw}$ laser. Left, simplified mechanism and reaction conditions. Right, kinetics of the $\mathrm{C}-\mathrm{Br}$ bond activation. Further mechanistic considerations can be found in Section S7.1 of the SI.

Having established that aqueous blue-to-UV upconversion can be exploited for the heterolytic cleavage of an unactivated $\mathrm{C}-\mathrm{Br}$ bond, we then investigated a more challenging reaction with potential wastewater treatment applications. The benzyltrimethylammonium cation is a common basic structure representing the substance class of quaternary ammonium compounds (QACs), which are large-scale industrial products being environmentally harmful. ${ }^{55}$ Previous photochemical strategies addressing the decomposition of QACs had to rely on UVC photons or even gamma rays for thermodynamic reasons. The only visible-light driven QAC decomposition, which pools the energy of two blue photons for the generation of hydrated electrons (reduction potential, $-2.9 \mathrm{~V} v s$. NHE), has been developed recently, ${ }^{37}$ but it requires very high photon fluxes per area on the order of $1 \mathrm{~kW} \mathrm{~cm}{ }^{-2}$. An UC-driven mechanism for the challenging reductive QAC decomposition in water would be a significant improvement, because such a mechanism would likely operate at light power densities as low as provided by conventional LEDs. Such an improvement could eventually initiate the development of environmental remediation applications on a larger scale.

${ }^{3}$ Irsppy with its excited-state oxidation potential of $-1.64 \mathrm{~V}$ vs. NHE does not induce the benzyltrimethylammonium cation degradation. ${ }^{37}$ Increasing the redox potential available for reductions by almost $1 \mathrm{~V}$ when using ${ }^{1}$ NPX* instead of ${ }^{3}$ Irsppy, we speculated that the reduction threshold of the pollutant might be reached. Upon direct excitation of NPX with UV light, we indeed observed a pronounced decrease of the persistent ammonium ion along with 
trimethylamine production (Fig. 6), providing evidence for the ${ }^{1}$ NPX* induced QAC degradation (see SI, Section S7.2). These observations and the promising blue-to-UV UC results of the preceding sections prompted us to study this challenging reaction with blue light under UC conditions. Our irradiation experiments employing Irsppy as sensitizer combined with either a diode laser (447 $\mathrm{nm}$ ) or a collimated LED $\left(455 \mathrm{~nm}\right.$ ) clearly point to ${ }^{1} \mathrm{NPX} *$ produced via UC as key species. Under optimized reaction conditions, we achieved complete degradation of $10 \mathrm{mM}$ of the QAC (in $3 \mathrm{~mL}$ of alkaline water) within $23 \mathrm{~h}$ of LED illumination (Fig. 6), whereas control experiments without NPX but otherwise identical conditions did not show any conversion. The NPX monoanion and the benzyltrimethylammonium cation form an aggregate with 1:1 stoichiometry and a very low aggregation constant in aqueous solution, as revealed by a Benesi-Hildebrand analysis ${ }^{56}$ (see SI, Section S7.2). The aggregates are still water-soluble and about $90 \%$ of all NPX and QAC molecules are present as free ions under our typical reaction conditions. Hence, this aggregation phenomenon does not negatively affect the ${ }^{1} \mathbf{N P X}$-driven QAC decomposition summarized in Fig. 6.

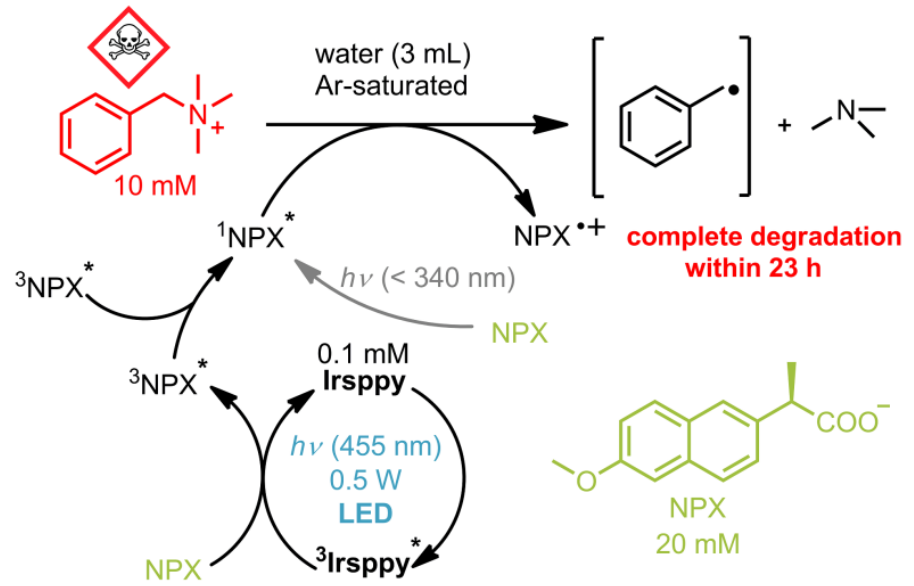

Figure 6. LED-driven decomposition of a QAC pollutant. Reductive degradation of benzyltrimethylammonium chloride in Ar-saturated $\mathrm{D}_{2} \mathrm{O}$ solution exploiting sTTA driven by a blue LED.

The results of this section on obtaining and exploiting unprecedented redox reactivities through UC in water provide an important proof-of-principle for the hidden potential of the sTTA mechanism, and might pave the way for more sustainable wastewater treatment or photoredox catalysis methodologies. 


\section{Conclusions}

Upconversion in the "greenest" solvent, neat water, via the sTTA mechanism is not merely a scientific curiosity but has the potential for valuable chemical applications, as has emerged from this study. Despite using an uncommon and very challenging solvent for sTTA, our investigations do not only set a new record for the most highly energetic emissive excited singlet states (3.9 eV) accessible with visible photons as energy input, but they also demonstrate photoredox applications with both synthetic and environmental remediation background. Novel watersoluble Ir sensitizers with unmatched properties formed the basis for that achievement, and these Ir complexes will most likely be useful for numerous subsequent sensitization and photoredox studies. The successful realization of visible-to-UV sTTA upconversion in aqueous solution might stimulate studies on hydrogen generation via water splitting, which inherently requires water-tolerance and high-energy photons, ${ }^{57}$ and it has the potential - after further optimization - to replace UV-light driven photoreactions frequently relying on mercury-based light sources. Our study thus provides a promising approach for more sustainability in photochemistry, $^{58}$ especially if earth-abundant ${ }^{59,60}$ or organic sensitizers ${ }^{61,62}$ could be incorporated into future aqueous UC systems.

\section{Supporting Information (SI)}

The Supporting Information is available free of charge at https://XXX.

General experimental details, synthetic protocols, characterization data, additional photochemical properties and spectroscopic results, control experiments, kinetic data, and application-related details.

\section{Corresponding Authors}

Oliver S. Wenger (oliver.wenger@unibas.ch)

Christoph Kerzig (christoph.kerzig@unibas.ch)

\section{Notes}

There are no competing interests to declare. 


\section{Acknowledgements}

Financial support provided by the Swiss National Science Foundation (grant number 200021_178760 and NCCR Molecular System Engineering) and by the German National Academy of Sciences Leopoldina (postdoctoral fellowship LPDS 2017-11) is gratefully acknowledged. C.K. thanks the Research Fund of the University of Basel for a Novartis University of Basel Excellence Scholarship for Life Sciences. K.B. gratefully acknowledges financial support from the European Research Council (ERC-2017-StG-757733) and the Knut and Alice Wallenberg Foundation (KAW 2017.0192).

\section{References}

(1) Parker, C. A.; Hatchard, C. G. Sensitised Anti-Stokes Delayed Fluorescence. Proc. Chem. Soc. 1962, 386-387.

(2) Singh-Rachford, T. N.; Castellano, F. N. Photon Upconversion Based on Sensitized Triplet-Triplet Annihilation. Coord. Chem. Rev. 2010, 254, 2560-2573.

(3) Gray, V.; Dzebo, D.; Abrahamsson, M.; Albinsson, B.; Moth-Poulsen, K. Triplet-Triplet Annihilation Photon-Upconversion: Towards Solar Energy Applications. Phys. Chem. Chem. Phys. 2014, 16, 10345-10352.

(4) Zhou, J.; Liu, Q.; Feng, W.; Sun, Y.; Li, F. Upconversion Luminescent Materials: Advances and Applications. Chem. Rev. 2015, 115, 395-465.

(5) Zhao, J.; Ji, S.; Guo, H. Triplet-Triplet Annihilation Based Upconversion: From Triplet Sensitizers and Triplet Acceptors to Upconversion Quantum Yields. RSC Adv. 2011, 1, 937-950.

(6) Manna, M. K.; Shokri, S.; Wiederrecht, G. P.; Gosztola, D. J.; Ayitou, A. J.-L. New Perspectives for Triplet-Triplet Annihilation Based Photon Upconversion Using All-Organic Energy Donor \& Acceptor Chromophores. Chem. Commun. 2018, 54, 5809-5818.

(7) Islangulov, R. R.; Castellano, F. N. Photochemical Upconversion: Anthracene Dimerization Sensitized to Visible Light by a Ru" Chromophore. Angew. Chem. Int. Ed. 2006, 45, 5957-5959.

(8) Majek, M.; Faltermeier, U.; Dick, B.; Pérez-Ruiz, R.; Jacobi von Wangelin, A. Application of Visibleto-UV Photon Upconversion to Photoredox Catalysis: The Activation of Aryl Bromides. Chem. Eur. J 2015, 21, 15496-15501.

(9) López-Calixto, C. G.; Liras, M.; de la Peña O'Shea, V. A.; Pérez-Ruiz, R. Synchronized Biphotonic Process Triggering C-C Coupling Catalytic Reactions. Appl. Catal. B Environ. 2018, 237, 18-23.

(10) Ravetz, B. D.; Pun, A. B.; Churchill, E. M.; Congreve, D. N.; Rovis, T.; Campos, L. M. Photoredox Catalysis Using Infrared Light via Triplet Fusion Upconversion. Nature 2019, 565, 343-346.

(11) Sasaki, Y.; Oshikawa, M.; Bharmoria, P.; Kouno, H.; Hayashi-Takagi, A.; Sato, M.; Ajioka, I.; Yanai, N.; Kimizuka, N. Near-Infrared Optogenetic Genome Engineering Based on Photon-Upconversion Hydrogels. Angew. Chem. Int. Ed. 2019, 58, 17827-17833.

(12) Askes, S. H. C.; Pomp, W.; Hopkins, S. L.; Kros, A.; Wu, S.; Schmidt, T.; Bonnet, S. Imaging Upconverting Polymersomes in Cancer Cells: Biocompatible Antioxidants Brighten Triplet-Triplet Annihilation Upconversion. Small 2016, 12, 5579-5590.

(13) Sanders, S. N.; Gangishetty, M. K.; Sfeir, M. Y.; Congreve, D. N. Photon Upconversion in Aqueous Nanodroplets. J. Am. Chem. Soc. 2019, 141, 9180-9184. 
(14) Tokunaga, A.; Uriarte, L. M.; Mutoh, K.; Fron, E.; Hofkens, J.; Sliwa, M.; Abe, J. Photochromic Reaction by Red Light via Triplet Fusion Upconversion. J. Am. Chem. Soc. 2019, 141, 17744-17753.

(15) Börjesson, K.; Dzebo, D.; Albinsson, B.; Moth-Poulsen, K. Photon Upconversion Facilitated Molecular Solar Energy Storage. J. Mater. Chem. A 2013, 1, 8521-8524.

(16) Nattestad, A.; Cheng, Y. Y.; MacQueen, R. W.; Schulze, T. F.; Thompson, F. W.; Mozer, A. J.; Fückel, B.; Khoury, T.; Crossley, M. J.; Lips, K.; Wallace, G. G.; Schmidt, T. W. Dye-Sensitized Solar Cell with Integrated Triplet-Triplet Annihilation Upconversion System. J. Phys. Chem. Lett. 2013, 4, 20732078.

(17) El Roz, K. A.; Castellano, F. N. Photochemical Upconversion in Water. Chem. Commun. 2017, 53, 11705-11708.

(18) Kerzig, C.; Wenger, O. S. Sensitized Triplet-Triplet Annihilation Upconversion in Water and Its Application to Photochemical Transformations. Chem. Sci. 2018, 9, 6670-6678.

(19) Singh-Rachford, T. N.; Castellano, F. N. Low Power Visible-to-UV Upconversion. J. Phys. Chem. A 2009, 113, 5912-5917.

(20) Yanai, N.; Kozue, M.; Amemori, S.; Kabe, R.; Adachi, C.; Kimizuka, N. Increased Vis-to-UV Upconversion Performance by Energy Level Matching between a TADF Donor and High Triplet Energy Acceptors. J. Mater. Chem. C 2016, 4, 6447-6451.

(21) Capello, C.; Fischer, U.; Hungerbühler, K. What Is a Green Solvent? A Comprehensive Framework for the Environmental Assessment of Solvents. Green Chem. 2007, 9, 927-934.

(22) Yu, H.; Ru, S.; Dai, G.; Zhai, Y.; Lin, H.; Han, S.; Wei, Y. An Efficient Iron(III)-Catalyzed Aerobic Oxidation of Aldehydes in Water for the Green Preparation of Carboxylic Acids. Angew. Chem. Int. Ed. 2017, 56, 3867-3871.

(23) Lippincott, D. J.; Linstadt, R. T. H.; Maser, M. R.; Gallou, F.; Lipshutz, B. H. Synthesis of Functionalized 1,3-Butadienes via Pd-Catalyzed Cross-Couplings of Substituted Allenic Esters in Water at Room Temperature. Org. Lett. 2018, 20, 4719-4722.

(24) Takale, B. S.; Thakore, R. R.; Handa, S.; Gallou, F.; Reilly, J.; Lipshutz, B. H. A New, Substituted Palladacycle for ppm Level Pd-Catalyzed Suzuki-Miyaura Cross Couplings in Water. Chem. Sci. 2019, 10, 8825-8831.

(25) Guo, X.; Okamoto, Y.; Schreier, M. R.; Ward, T. R.; Wenger, O. S. Enantioselective Synthesis of Amines by Combining Photoredox and Enzymatic Catalysis in a Cyclic Reaction Network. Chem. Sci. 2018, 9, 5052-5056.

(26) Giedyk, M.; Narobe, R.; Weiß, S.; Touraud, D.; Kunz, W.; König, B. Photocatalytic Activation of Alkyl Chlorides by Assembly-Promoted Single Electron Transfer in Microheterogeneous Solutions. Nat. Catal. 2020, 3, 40-47.

(27) Naumann, R.; Goez, M. How the Sustainable Solvent Water Unleashes the Photoredox Catalytic Potential of Ruthenium Polypyridyl Complexes for Pinacol Couplings. Green Chem. 2019, 21, 4470-4474.

(28) Fan, C.; Wei, L.; Niu, T.; Rao, M.; Cheng, G.; Chruma, J. J.; Wu, W.; Yang, C. Efficient Triplet-Triplet Annihilation Upconversion with an Anti-Stokes Shift of $1.08 \mathrm{eV}$ Achieved by Chemically Tuning Sensitizers. J. Am. Chem. Soc. 2019, 141, 15070-15077.

(29) Nishimura, N.; Gray, V.; Allardice, J. R.; Zhang, Z.; Pershin, A.; Beljonne, D.; Rao, A. Photon Upconversion from Near-Infrared to Blue Light with TIPS-Anthracene as an Efficient Triplet-Triplet Annihilator. ACS Mater. Lett. 2019, 1, 660-664.

(30) Chen, W.; Song, F.; Tang, S.; Hong, G.; Wu, Y.; Peng, X. Red-to-Blue Photon up-Conversion with High Efficiency Based on a TADF Fluorescein Derivative. Chem. Commun. 2019, 55, 4375-4378.

(31) Li, L.; Zeng, Y.; Chen, J.; Yu, T.; Hu, R.; Yang, G.; Li, Y. Thermally Activated Delayed Fluorescence via Triplet Fusion. J. Phys. Chem. Lett. 2019, 10, 6239-6245.

(32) Glaser, F.; Kerzig, C.; Wenger, O. S. Multi-Photon Excitation in Photoredox Catalysis: Concepts, Applications, Methods. Angew. Chem. Int. Ed. 2020. https://doi.org/10.1002/anie.201915762. 
(33) Barawi, M.; Fresno, F.; Pérez-Ruiz, R.; de la Peña O’Shea, V. A. Photoelectrochemical Hydrogen Evolution Driven by Visible-to-Ultraviolet Photon Upconversion. ACS Appl. Energy Mater. 2019, 2, 207-211.

(34) He, S.; Luo, X.; Liu, X.; Li, Y.; Wu, K. Visible-to-Ultraviolet Upconversion Efficiency above $10 \%$ Sensitized by Quantum-Confined Perovskite Nanocrystals. J. Phys. Chem. Lett. 2019, 10, 50365040.

(35) Hisamitsu, S.; Miyano, J.; Okumura, K.; Hui, J. K.; Yanai, N.; Kimizuka, N. Visible-to-UV Photon Upconversion in Nanostructured Chromophoric lonic Liquids. ChemistryOpen 2020, 9, 14-17.

(36) Kawashima, Y.; Kouno, H.; Orihashi, K.; Nishimura, K.; Yanai, N.; Kimizuka, N. Visible-to-UV Photon Upconversion in Air-Saturated Water by Multicomponent Co-Assembly. Mol. Syst. Des. Eng. 2020. https://doi.org/10.1039/DOME00003E.

(37) Kerzig, C.; Guo, X.; Wenger, O. S. Unexpected Hydrated Electron Source for Preparative VisibleLight Driven Photoredox Catalysis. J. Am. Chem. Soc. 2019, 141, 2122-2127.

(38) Naumann, R.; Kerzig, C.; Goez, M. Laboratory-Scale Photoredox Catalysis Using Hydrated Electrons Sustainably Generated with a Single Green Laser. Chem. Sci. 2017, 8, 7510-7520.

(39) Kerzig, C.; Wenger, O. S. Reactivity Control of a Photocatalytic System by Changing the Light Intensity. Chem. Sci. 2019, 10, 11023-11029.

(40) Teegardin, K.; Day, J. I.; Chan, J.; Weaver, J. Advances in Photocatalysis: A Microreview of Visible Light Mediated Ruthenium and Iridium Catalyzed Organic Transformations. Org. Process Res. Dev. 2016, 20, 1156-1163.

(41) Kerzig, C.; Goez, M. Highly Efficient Green-Light Ionization of an Aryl Radical Anion: Key Step in a Catalytic Cycle of Electron Formation. Phys. Chem. Chem. Phys. 2014, 16, 25342-25349.

(42) Ye, C.; Gray, V.; Mårtensson, J.; Börjesson, K. Annihilation Versus Excimer Formation by the Triplet Pair in Triplet-Triplet Annihilation Photon Upconversion. J. Am. Chem. Soc. 2019, 141, 9578-9584.

(43) Montalti, M.; Credi, A.; Prodi, L.; Gandolfi, M. T. Handbook of Photochemistry, 3rd ed.; CRC/Taylor \& Francis: Boca Raton, 2006.

(44) Arias-Rotondo, D. M.; McCusker, J. K. The Photophysics of Photoredox Catalysis: A Roadmap for Catalyst Design. Chem. Soc. Rev. 2016, 45, 5803-5820.

(45) Mills, I. N.; Porras, J. A.; Bernhard, S. Judicious Design of Cationic, Cyclometalated Ir(III) Complexes for Photochemical Energy Conversion and Optoelectronics. Acc. Chem. Res. 2018, 51, 352-364.

(46) Strieth-Kalthoff, F.; James, M. J.; Teders, M.; Pitzer, L.; Glorius, F. Energy Transfer Catalysis Mediated by Visible Light: Principles, Applications, Directions. Chem. Soc. Rev. 2018, 47, 71907202.

(47) Gray, V.; Xia, P.; Huang, Z.; Moses, E.; Fast, A.; Fishman, D. A.; Vullev, V. I.; Abrahamsson, M.; Moth-Poulsen, K.; Lee Tang, M. CdS/ZnS Core-Shell Nanocrystal Photosensitizers for Visible to UV Upconversion. Chem. Sci. 2017, 8, 5488-5496.

(48) Luo, X.; Han, Y.; Chen, Z.; Li, Y.; Liang, G.; Liu, X.; Ding, T.; Nie, C.; Wang, M.; Castellano, F. N.; Wu, K. Mechanisms of Triplet Energy Transfer across the Inorganic Nanocrystal/Organic Molecule Interface. Nat. Commun. 2020, 11, 28.

(49) Liang, R.; Sun, S.-S.; Huang, G.; Li, M.-D. Unveiling the Photophysical and Photochemical Reaction Process of Naproxen via Ultrafast Femtosecond to Nanosecond Laser Flash Photolysis. Chem. Res. Toxicol. 2019, 32, 613-620.

(50) Fonseca, W. T.; Santos, R. F.; Alves, J. N.; Ribeiro, S. D.; Takeuchi, R. M.; Santos, A. L.; Assunção, R. M. N.; Filho, G. R.; Muñoz, R. A. A. Square-Wave Voltammetry as Analytical Tool for Real-Time Study of Controlled Naproxen Releasing from Cellulose Derivative Materials. Electroanalysis 2015, $27,1847-1854$.

(51) Isokuortti, J.; Allu, S. R.; Efimov, A.; Vuorimaa-Laukkanen, E.; Tkachenko, N. V.; Vinogradov, S. A.; Laaksonen, T.; Durandin, N. A. Endothermic and Exothermic Energy Transfer Made Equally Efficient for Triplet-Triplet Annihilation Upconversion. J. Phys. Chem. Lett. 2020, 11, 318-324. 
(52) Perego, J.; Pedrini, J.; Bezuidenhout, C. X.; Sozzani, P. E.; Meinardi, F.; Bracco, S.; Comotti, A.; Monguzzi, A. Engineering Porous Emitting Framework Nanoparticles with Integrated Sensitizers for Low-Power Photon Upconversion by Triplet Fusion. Adv. Mater. 2019, 31, 1903309.

(53) Haefele, A.; Blumhoff, J.; Khnayzer, R. S.; Castellano, F. N. Getting to the (Square) Root of the Problem: How to Make Noncoherent Pumped Upconversion Linear. J. Phys. Chem. Lett. 2012, 3, 299-303.

(54) Wei, Y.; Zheng, M.; Chen, L.; Zhou, X.; Liu, S. Near-Infrared to Violet Triplet-Triplet Annihilation Fluorescence Upconversion of Os(II) Complexes by Strong Spin-Forbidden Transition. Dalton Trans. 2019, 48, 11763-11771.

(55) Tezel, U.; Pavlostathis, S. G. Quaternary Ammonium Disinfectants: Microbial Adaptation, Degradation and Ecology. Curr. Opin. Biotechnol. 2015, 33, 296-304.

(56) Benesi, H. A.; Hildebrand, J. H. A Spectrophotometric Investigation of the Interaction of lodine with Aromatic Hydrocarbons. J. Am. Chem. Soc. 1949, 71, 2703-2707.

(57) Kudo, A.; Miseki, Y. Heterogeneous Photocatalyst Materials for Water Splitting. Chem. Soc. Rev. 2009, 38, 253-278.

(58) Bochet, C. G. On the Sustainability of Photochemical Reactions. Chimia 2019, 73, 720-723.

(59) Förster, C.; Heinze, K. Photophysics and Photochemistry with Earth-Abundant Metals Fundamentals and Concepts. Chem. Soc. Rev. 2020, 49, 1057-1070.

(60) Wenger, O. S. Photoactive Complexes with Earth-Abundant Metals. J. Am. Chem. Soc. 2018, 140, 13522-13533.

(61) Wu, W.; Guo, H.; Wu, W.; Ji, S.; Zhao, J. Organic Triplet Sensitizer Library Derived from a Single Chromophore (BODIPY) with Long-Lived Triplet Excited State for Triplet-Triplet Annihilation Based Upconversion. J. Org. Chem. 2011, 76, 7056-7064.

(62) Du, Y.; Pearson, R. M.; Lim, C.-H.; Sartor, S. M.; Ryan, M. D.; Yang, H.; Damrauer, N. H.; Miyake, G. M. Strongly Reducing, Visible-Light Organic Photoredox Catalysts as Sustainable Alternatives to Precious Metals. Chem. - Eur. J. 2017, 23, 10962-10968.

\section{Table of Contents (TOC) Graphic}

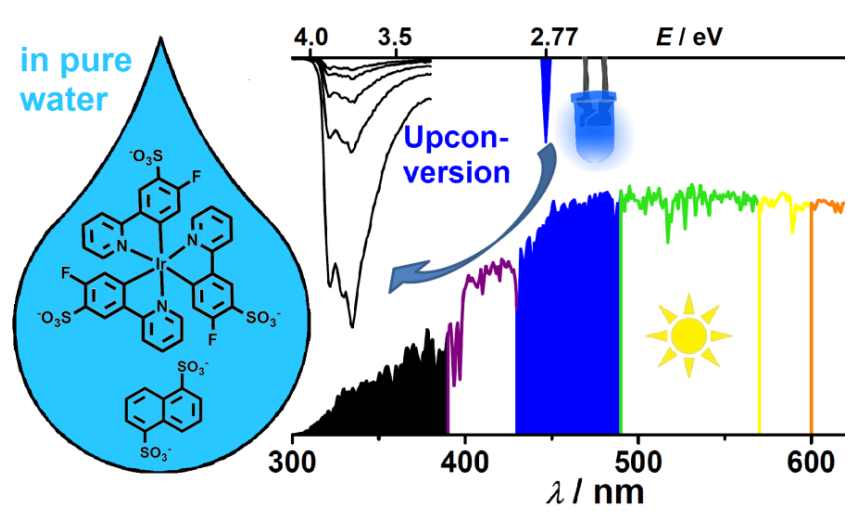

